International Journal of Pure and Applied Mathematics

Volume 106 No. $3 \quad 2016,841-849$

ISSN: $1311-8080$ (printed version); ISSN: 1314-3395 (on-line version)

url: http://www.ijpam.eu

doi: 10.12732/ijpam.v106i3.10

\title{
DIRECTLY SOLVING SECOND ORDER LINEAR BOUNDARY VALUE PROBLEMS OF ORDINARY DIFFERENTIAL EQUATIONS
}

\author{
Ra'ft Abdelrahim ${ }^{1}$, Z. Omar ${ }^{2}$ \\ ${ }^{1,2}$ Department of Mathematics \\ School of Quantitative Sciences \\ College of Art and Sciences \\ Univeristi Utara Malaysia \\ MALAYSIA
}

\begin{abstract}
In this article, boundary value problems of second order linear ordinary differential equations are solved directly using hybrid block one-step method. Power series of order six is adopted as basis function to derive this method through collocation and interpolation approach. Shooting method is employed to transform boundary value problem into initial value problem. Then hybrid block one step is used to approximate the solution. The performance of the new method is shown by solving some boundary value problems examined by previous methods.
\end{abstract}

AMS Subject Classification: 35J25, 35Q60, 65L06, 65L05

Key Words: hybrid one step, block method, second order boundary value problem, three off step points, shooting method

\section{Introduction}

Mathematical models are expressed by boundary value problem (BVPs) and initial value problem (IVPs) of ordinary differential equations in order to help

Received: December 14, 2015

(c) 2016 Academic Publications, Ltd.

Published: February 27, 2016

url: www.acadpubl.eu

$\S_{\text {Correspondence author }}$ 
in understanding the physical phenomena. BVPs have long been a wide area of study because of their vast application in sciences such as physics, engineering, biology and chemistry. In general, it is not easy to solve these types of problems analytically [1]. Several numerical methods have been derived to approximate the solutions for BVPs.

According to [7], the using of spline methods for solving BVPs was initially investigated by Bickley in 1968. In the same work, Adomian Decomposition Method (ADM) has also been widely used for solving BVPs ( see [6] and [9]). In 2002, [8] proposed different finite numerical method for solving two-point BVPs. Recently, [10] presented three-points block one-step method for solving second order linear Dirichlet and Neumann BVPs directly. However, these methods have dealt with some difficulties for solving BVPs which leads to inefficiently in term of error.

Our focus in this paper is to derive new one step hybrid block method with three off step points for solving second order linear BVPs. It worth to highlight that, one step hybrid block method combines the advantages of block and hybrid methods( see [2], [5]) which overcomes the zero stability barrier in linear multistep method [3].

\section{Derivation of Method}

The following second order two points linear (BVPs) of the form:

$$
\begin{array}{r}
y^{\prime \prime}=p(x) y^{\prime}+q(x) y+r(x), \quad q(x) \geq 0, \quad x \in[a, b] . \\
y(a)=\alpha, \quad y(b)=\lambda,
\end{array}
$$

is considered.

In our strategy, the first step is employing shooting method to transform equation (1) into two IVPs as below:

$$
\begin{array}{rrr}
y_{1}^{\prime \prime}=p(x) y_{1}^{\prime}+q(x) y_{1}+r(x), & y_{1}(a)=\alpha, & y_{1}^{\prime}(a)=0 \\
y_{2}^{\prime \prime}=p(x) y_{2}^{\prime}+q(x) y_{2}, & y_{2}(a)=0, & y_{2}^{\prime}(a)=1 .
\end{array}
$$

Then, the developed one step hybrid block method is used to solve (2) directly. Finally, the approximate solution for(1) is obtained as following:

$$
y(x)=y_{1}(x)+\frac{\lambda-y_{1}(b)}{y_{2}(b)} y_{2}(x) .
$$


In order to determine $y_{1}(x)$ and $y_{2}(x)$, a power series of the form

$$
y(x)=\sum_{i=0}^{v+m-1} a_{i}\left(\frac{x-x_{n}}{h}\right)^{i} \cdot x \in\left[x_{n}, x_{n+1}\right]
$$

is considered for approximation solution of (2), where $n=0,1,2, \ldots, N-1$, $v=2$ represents the number of interpolation points, $m=5$ denotes the number of collocation points, $h=x_{n}-x_{n-1}$ and $[a, b]$ is divided as following $a=x_{0}<$ $x_{1}<\ldots<x_{N-1}<x_{N}=b$.

Initially, equation (1) is interpolated at points $x_{n+\frac{1}{3}}, x_{n+\frac{2}{3}}$ and its second derivative is collocated at all points i.e $x_{n}, x_{n+\frac{1}{4}}, x_{n+\frac{1}{3}}, x_{n+\frac{2}{3}}$ and $x_{n+1}$. This will produce the following system of equations

$$
\left(\begin{array}{ccccccc}
1 & \frac{1}{3} & \frac{1}{9} & \frac{1}{27} & \frac{1}{81} & \frac{1}{243} & \frac{1}{729} \\
1 & \frac{2}{3} & \frac{4}{9} & \frac{8}{27} & \frac{16}{81} & \frac{32}{243} & \frac{64}{729} \\
0 & 0 & \frac{2}{h^{2}} & 0 & 0 & 0 & 0 \\
0 & 0 & \frac{2}{h^{2}} & \frac{3}{\left(2 h^{2}\right)} & \frac{3}{\left(4 h^{2}\right)} & \frac{5}{\left(16 h^{2}\right)} & \frac{15}{\left(128 h^{2}\right)} \\
0 & 0 & \frac{2}{h^{2}} & \frac{2}{h^{2}} & \frac{4}{\left(3 h^{2}\right)} & \frac{20}{\left(27 h^{2}\right)} & \frac{10}{\left(27 h^{2}\right)} \\
0 & 0 & \frac{2}{h^{2}} & \frac{4}{h^{2}} & \frac{16}{\left(3 h^{2}\right)} & \frac{160}{\left(27 h^{2}\right)} & \frac{160}{\left(27 h^{2}\right)} \\
0 & 0 & \frac{2}{h^{2}} & \frac{6}{h^{2}} & \frac{12}{h^{2}} & \frac{20}{h^{2}} & \frac{30}{h^{2}}
\end{array}\right)\left(\begin{array}{c}
a_{0} \\
a_{1} \\
a_{2} \\
a_{3} \\
a_{4} \\
a_{5} \\
a_{6}
\end{array}\right)=\left(\begin{array}{c}
y_{n+\frac{1}{3}} \\
y_{n+\frac{2}{3}} \\
f_{n} \\
f_{n+\frac{1}{4}} \\
f_{n+\frac{1}{3}} \\
f_{n+\frac{2}{3}} \\
f_{n+1}
\end{array}\right)
$$

Gaussian elimination method is used in (4) to find the values of $a_{i}{ }^{\prime} s, i=0(1) 6$ and then substituted into equation (1)to obtain a continuous linear multistep method of the form:

$$
y(x)=\sum_{i=\frac{1}{3}, \frac{2}{3}} \alpha_{i}(x) y_{n+s_{i}}+\sum_{i=0}^{1} \beta_{i}(x) f_{n+i}+\sum_{i=\frac{1}{4}, \frac{1}{3}, \frac{2}{3}} \beta_{i} f_{n+i} .
$$

The first derivative of equation (5) are given by

$$
y^{\prime}(x)=\sum_{i=\frac{1}{3}, \frac{2}{3}} \frac{d}{d x} \alpha_{i}(x) y_{n+s_{i}}+\sum_{i=0}^{1} \frac{d}{d x} \beta_{i}(x) f_{n+i}+\sum_{i=\frac{1}{4}, \frac{1}{3}, \frac{2}{3}} \frac{d}{d x}(x) \beta_{i} f_{n+i}
$$

where

$$
\begin{aligned}
& \alpha_{\frac{1}{3}}=\left(2-\frac{\left(3\left(x-x_{n}\right)\right)}{h}\right) \\
& \alpha_{\frac{2}{3}}=\left(\frac{\left(3\left(x-x_{n}\right)\right)}{h}-1\right)
\end{aligned}
$$




$$
\begin{aligned}
\beta_{0}= & \frac{\left(x-x_{n}\right)^{2}}{2}-\frac{\left(19\left(x-x_{n}\right)^{3}\right)}{(12 h)}+\frac{\left(31\left(x-x_{n}\right)^{4}\right)}{\left(12 h^{2}\right)}+\frac{\left(11 h^{2}\right)}{1620} \\
& +\frac{\left(3\left(x-x_{n}\right)^{6}\right)}{\left(5 h^{4}\right)}-\frac{\left(91 h\left(x-x_{n}\right)\right)}{1080}-\frac{\left(81\left(x-x_{n}\right)^{5}\right)}{\left(40 h^{3}\right)} \\
\beta_{\frac{1}{4}}= & \frac{\left(256\left(x-x_{n}\right)^{3}\right)}{(45 h)}-\frac{\left(704\left(x-x_{n}\right)^{4}\right)}{\left(45 h^{2}\right)}+\frac{\left(384\left(x-x_{n}\right)^{5}\right)}{\left(25 h^{3}\right)} \\
& -\frac{\left(64 h\left(x-x_{n}\right)\right)}{225}+\frac{\left(128 h^{2}\right)}{6075}-\frac{\left(128\left(x-x_{n}\right)^{6}\right)}{\left(25 h^{4}\right)} \\
\beta_{\frac{1}{3}}= & \frac{\left(117\left(x-x_{n}\right)^{4}\right)}{\left(8 h^{2}\right)}-\frac{\left(9\left(x-x_{n}\right)^{3}\right)}{(2 h)}-\frac{\left(621\left(x-x_{n}\right)^{5}\right)}{\left(40 h^{3}\right)} \\
& -\frac{\left(h\left(x-x_{n}\right)\right)}{12}+\frac{\left(19 h^{2}\right)}{270}+\frac{\left(27\left(x-x_{n}\right)^{6}\right)}{\left(5 h^{4}\right)} \\
\beta_{\frac{2}{3}}= & \left(\frac{\left(9\left(x-x_{n}\right)^{3}\right)}{(20 h)}-\frac{\left(9\left(x-x_{n}\right)^{4}\right)}{\left(5 h^{2}\right)}+\frac{\left(513\left(x-x_{n}\right)^{5}\right)}{\left(200 h^{3}\right)}\right. \\
& -\frac{\left(31 h\left(x-x_{n}\right)\right)}{600}+\frac{\left(37 h^{2}\right)}{2700}-\frac{\left(27\left(x-x_{n}\right)^{6}\right)}{\left(25 h^{4}\right)} \\
\beta_{1}= & \frac{\left(17\left(x-x_{n}\right)^{4}\right)}{\left(72 h^{2}\right)}-\frac{\left(x-x_{n}\right)^{3}}{(18 h)}-\frac{\left(3\left(x-x_{n}\right)^{5}\right)}{\left(8 h^{3}\right)} \\
+ & \frac{\left(h\left(x-x_{n}\right)\right)}{270}-\frac{h^{2}}{1215}+\frac{\left(x-x_{n}\right)^{6}}{\left(5 h^{4}\right)}
\end{aligned}
$$

Equation(5)is evaluated at the non-interpolating point i.e $x_{n+\frac{1}{4}}, x_{n+1}$ and equation (6) are evaluated at all points to produce the discrete schemes and its derivatives. The discrete scheme and its derivatives are combined in matrix of the form block as below

$$
A^{[3]_{2}} Y_{m}^{[3]_{2}}=B^{[3]_{2}} R_{1}^{[3]_{2}}+h^{2} D^{[3]_{2}} R_{2}^{[3]_{2}}+h^{2} E^{[3]_{2}} R_{3}^{[3]_{2}},
$$

where

$$
A^{[3]_{2}}=\left(\begin{array}{cccccccc}
0 & -2 & 1 & 0 & 0 & 0 & 0 & 0 \\
1 & \frac{-5}{4} & \frac{1}{4} & 0 & 0 & 0 & 0 & 0 \\
0 & 1 & -2 & 1 & 0 & 0 & 0 & 0 \\
0 & \frac{3}{h} & \frac{-3}{h} & 0 & 0 & 0 & 0 & 0 \\
0 & \frac{3}{h} & \frac{-3}{h} & 0 & 1 & 0 & 0 & 0 \\
0 & \frac{3}{h} & \frac{-3}{h} & 0 & 0 & 1 & 0 & 0 \\
0 & \frac{3}{h} & \frac{-3}{h} & 0 & 0 & 0 & 1 & 0 \\
0 & \frac{3}{h} & \frac{-3}{h} & 0 & 0 & 0 & 0 & 1
\end{array}\right), \quad Y_{m}^{[3]_{2}}=\left(\begin{array}{c}
y_{n+\frac{1}{4}} \\
y_{n+\frac{1}{3}} \\
y_{n+\frac{2}{3}} \\
y_{n+1}
\end{array}\right)
$$




$$
\begin{aligned}
& B^{[3]_{2}}=\left(\begin{array}{cc}
-1 & 0 \\
0 & 0 \\
0 & 0 \\
0 & -1 \\
0 & 0 \\
0 & 0 \\
0 & 0 \\
0 & 0
\end{array}\right), R_{1}^{[3]_{2}}\left(\begin{array}{c}
y_{n} \\
y_{n}^{\prime}
\end{array}\right), D^{[3]_{2}}=\left(\begin{array}{c}
\frac{\left(11 h^{2}\right)}{1620} \\
\frac{\left(329 h^{2}\right)}{663552} \\
\frac{-h^{2}}{405} \\
\frac{(-91 h)}{1080} \\
\frac{(-1579 h)}{276480} \\
\frac{-h}{162} \\
\frac{(23 h)}{3240} \\
\frac{(-7 h)}{270}
\end{array}\right), R_{2}^{[3]_{3}}\left(f_{n}\right), \\
& E^{[3]_{2}}=\left(\begin{array}{cccc}
\frac{\left(299 h^{2}\right)}{7200} & \frac{-\left(1107 h^{2}\right)}{40960} & \frac{\left(459 h^{2}\right)}{204800} & \frac{-\left(97 h^{2}\right)}{368640} \\
\frac{\left(448 h^{2}\right)}{6075} & \frac{-\left(23 h^{2}\right)}{540} & \frac{\left(19 h^{2}\right)}{5400} & \frac{-h^{2}}{2430} \\
\frac{\left(1024 h^{2}\right)}{6075} & \frac{-\left(2 h^{2}\right)}{135} & \frac{\left(14 h^{2}\right)}{675} & \frac{-\left(2 h^{2}\right)}{1215} \\
\frac{\left(64 h^{2}\right)}{225} & 0 & \frac{\left(27 h^{2}\right)}{200} & \frac{h^{2}}{180} \\
\frac{(323 h)}{900} & \frac{-(2061 h)}{10240} & \frac{(801 h)}{51200} & \frac{-(167 h)}{92160} \\
\frac{(2432 h)}{6075} & \frac{-(19 h)}{120} & \frac{(3 h)}{200} & \frac{-(17 h)}{9720} \\
\frac{(1024 h)}{6075} & \frac{(4 h)}{15} & \frac{(11 h)}{75} & \frac{-(8 h)}{1215} \\
\frac{(128 h)}{225} & \frac{-(9 h)}{40} & \frac{(99 h)}{200} & \frac{(37 h)}{360}
\end{array}\right) \text { and } R_{3}^{[3]_{3}}=\left(\begin{array}{c}
f_{n+\frac{1}{4}} \\
f_{n+\frac{1}{3}} \\
f_{n+\frac{2}{3}} \\
f_{n+1}
\end{array}\right)
\end{aligned}
$$

Multiplying Equation (7) by $\left(A^{[3]_{2}}\right)^{-1}$ produces the following hybrid block method

$$
{ }^{[3]_{2}} Y_{m}^{[3]_{2}}=\bar{B}^{[3]_{3}} R_{1}^{[3]_{3}}++h^{2} \bar{D}^{[3]_{2}} R_{2}^{[3]_{2}}+h^{2} \bar{E}^{[3]_{2}} R_{3}^{[3]_{2}}
$$

where

$$
I^{[3]_{2}}=\left(\begin{array}{cccc}
1 & 0 & 0 & 0 \\
0 & 1 & 0 & 0 \\
0 & 0 & 1 & 0 \\
0 & 0 & 0 & 1
\end{array}\right), \quad \bar{B}^{[3]_{2}}=\left(\begin{array}{cc}
1 & \frac{h}{4} \\
1 & \frac{h}{3} \\
1 & \frac{(2 h)}{3} \\
1 & h \\
0 & 1 \\
0 & 1 \\
0 & 1 \\
0 & 1
\end{array}\right), \quad \bar{D}^{[3]_{2}}=\left(\begin{array}{c}
\frac{\left(121 h^{2}\right)}{8192} \\
\frac{\left(23 h^{2}\right)}{1080} \\
\frac{\left(4 h^{2}\right)}{81} \\
\frac{\left(3 h^{2}\right)}{40} \\
\frac{(2413 h)}{30720} \\
\frac{(253 h)}{3240} \\
\frac{(37 h)}{405} \\
\frac{(7 h)}{120}
\end{array}\right),
$$




$$
\bar{E}^{[3]_{2}}=\left(\begin{array}{cccc}
\frac{3 h^{2}}{128} & -\frac{47 h^{2}}{3840} & \frac{29 h^{2}}{5760} & -\frac{7 h^{2}}{7680} \\
\frac{h^{2}}{10} & \frac{-h^{2}}{48} & \frac{h^{2}}{90} & \frac{-h^{2}}{480} \\
\frac{117 h^{2}}{640} & \frac{27 h^{2}}{1280} & \frac{3 h^{2}}{128} & -\frac{9 h^{2}}{2560} \\
\frac{4 h^{2}}{15} & \frac{h^{2}}{15} & \frac{\left.4 h^{2}\right)}{45} & 0 \\
\frac{323 h}{1440} & \frac{-11 h}{120} & \frac{53 h}{1440} & -\frac{19 h}{2880} \\
\frac{31 h}{90} & \frac{h}{15} & \frac{h}{90} & \frac{-h}{360} \\
\frac{51 h}{160} & \frac{9 h}{40} & \frac{21 h}{160} & -\frac{3 h}{320} \\
\frac{16 h}{45} & \frac{2 h}{15} & \frac{16 h}{45} & \frac{7 h}{90}
\end{array}\right)
$$

\subsubsection{Numerical Results}

In order to confirm the performance of the new one step hybrid block method, the following three second linear BVPS were solved and compared with existing methods in [6], [7] and [10] as demonstrated in Tables 1-3.

The notation below are used in the tables:

MAXE maximum error of the computed solution

EAD extended adomian decomposition method in [6]

3BVP direct three-point block one-step method in [10]

ECBIM extended cubic b-spline method minimizing using

Newton's method in [7]

OSHBT implementation of the one step hybrid block method with three off-step points.

\section{Problem 1.}

$$
y^{\prime \prime}(x)=y(x)+\cos (x), \quad y(0)=0, \quad y(1)=1, \quad x \in[0,1] .
$$

Exact solution:

$$
\begin{aligned}
y(x)= & \frac{-3 \cosh (1)+\sinh (1)+\cos (1)+2}{\sinh (1)} e^{x} \\
& +\frac{3 \cosh (1)+3 \sinh (1)-\cos (1)-2}{\sinh (1)} e^{-x} \\
& +\frac{\cos (x)}{2} .
\end{aligned}
$$

\section{Problem 2.}

$$
y^{\prime \prime}(x)=y^{\prime}(x)+e^{x-1}-1, \quad y(0)=0, \quad y(1)=1 .
$$

Exact solution:

$$
y(x)=x\left(1-e^{x-1}\right) .
$$




\begin{tabular}{||cccc||}
\hline$x$ & MAXE 3BVP [ 5] & MAXE EAD [13] & MAXE OSHBT \\
$\frac{2}{8}$ & $2.22 e^{-7}$ & $4.37 e^{-7}$ & $7.69 e^{-11}$ \\
$\frac{3}{8}$ & $3.20 e^{-7}$ & $8.07 e^{-7}$ & $1.49 e^{-10}$ \\
$\frac{1}{8}$ & $3.12 e^{-7}$ & $1.05 e^{-6}$ & $2.18 e^{-10}$ \\
$\frac{5}{8}$ & $2.90 e^{-7}$ & $1.14 e^{-6}$ & $2.85 e^{-10}$ \\
$\frac{6}{8}$ & $2.65 e^{-7}$ & $1.05 e^{-6}$ & $3.50 e^{-10}$ \\
$\frac{7}{8}$ & $1.30 e^{-7}$ & $8.07 e^{-7}$ & $4.16 e^{-10}$ \\
\hline
\end{tabular}

Table 1: Comparison of the new method with [6] and [10], solving Problem 1 with $h=0.125$

\section{Problem 3.}

$y^{\prime \prime}(x)=(-x-1) y^{\prime}(x)+2 y(x)+\left(1-x^{2}\right) e^{-x}, \quad y(0)=-1, \quad y(1)=0, \quad x \in[0,1]$.

Exact solution

$$
y(x)=x\left(1-e^{x-1}\right) .
$$

\section{Conclusion}

High accurate one step hybrid block method for solving second order linear Boundary value problem directly has been successfully developed. The new method outperformed the existing methods when solving the same BVPs of second order ODEs directly.

\section{References}

[1] T. A. Anake, Continuous implicit hybrid one-step methods for the solution of initial value problems of general second-order ordinary differential equations (Unpublished doctoral dissertation). Covenant University,2011 


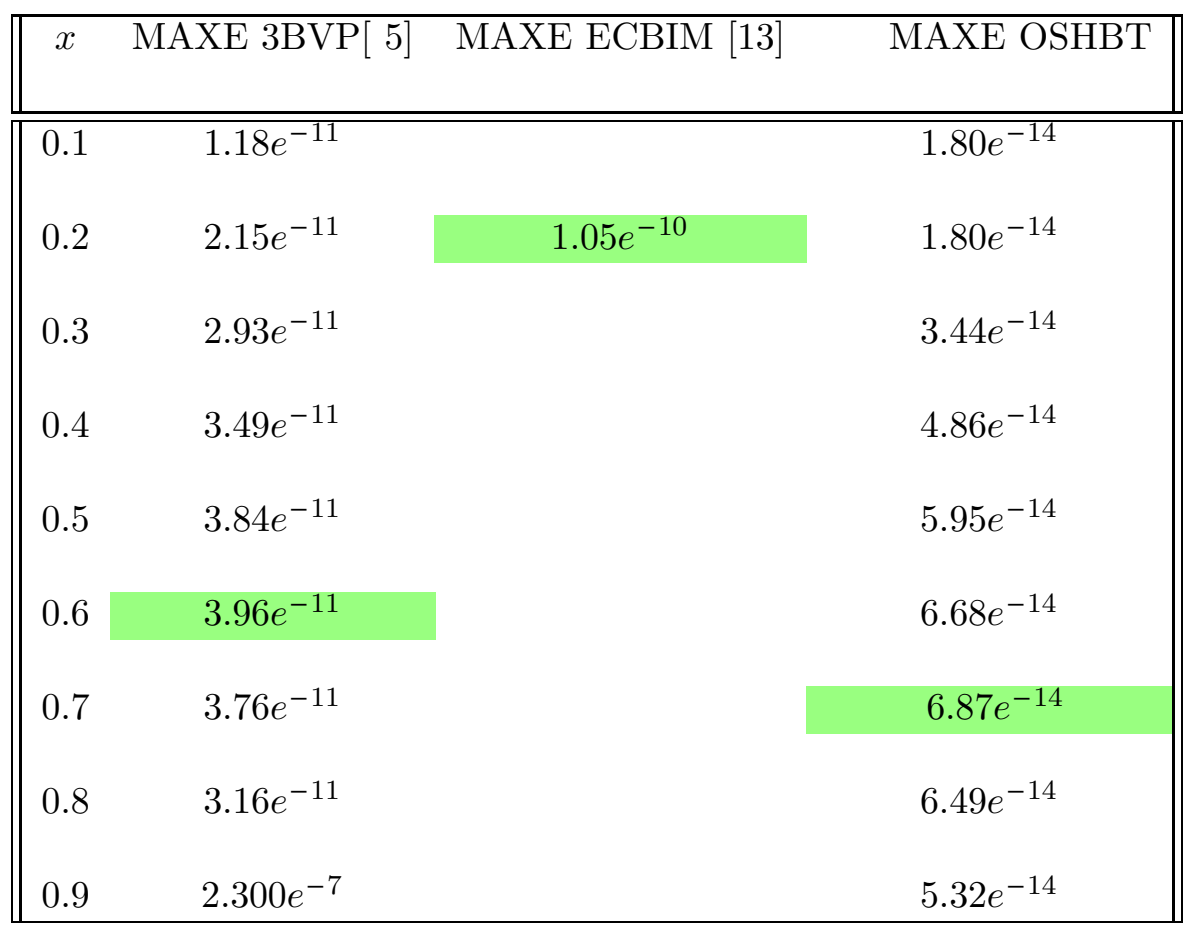

Table 2: Comparison of the new method with [10] and [7], solving Problem 2 with $h=0.01$

[2] A. Sagir, An accurate computation of block hybrid method for solving stiff ordinary differential equations, Journal of Mathematics,4,2012,pp. 18-21.

[3] J. D. Lambert, Computational Methods in Ordinary Differential Equation", John Wiley and Sons Inc, London,1973.

[4] D. O. Awoyemi, A P-stable Linear Multistep Method for Solving Third Order Ordinary Differential Equation, Inter. J. Computer Math, 8, 2003,pp. 985-991.

[5] Z.Omar and Ra'ft, Abdelrahim, Developing a Single Step Hybrid Block Method with Generalized Three Off -step Points for the Direct Solution of Second Order Ordinary Differential Equations, International Journal of Mathematical Analysis, 9,46, 2015, pp.22572272 .

[6] J. Bongsoo,Two-point boundary value problems by the extended Adomian decomposition method", Journal of Computational and Applied Mathematics, 219,1,2008, pp. 253-262.

[7] N. A. Hamid, A. A. Majid and A. I. Ismail, Extended cubic B-spline Method for Linear Two-Point Boundary Value Problems, Sains Malaysiana, 40,11,2011,pp. 1285-1290

[8] Q. Fang,T. Tsuchiya, and T.Yamamoto,Finite difference, finite element and finite volume methods applied to two-point boundary value problems, Journal of Computational and Applied Mathematics,139,1,2002,pp. 9-19 


\begin{tabular}{||llll|}
\hline$x$ & EXACT SOLUTION & COMPUTED SOLUTION & ERROR \\
\hline 0.1 & -0.814353676232363650 & -0.814353676232326460 & $3.719247 e^{-14}$ \\
0.2 & -0.654984602462385430 & -0.654984602462323600 & $6.195044 e^{-14}$ \\
0.3 & -0.518572754477202410 & -0.518572754477126700 & $7.593925 e^{-14}$ \\
0.4 & -0.402192027621383400 & -0.402192027621303240 & $8.038015 e^{-14}$ \\
0.5 & -0.303265329856316550 & -0.303265329856237660 & $7.932544 e^{-14}$ \\
0.6 & -0.219524654437610350 & -0.219524654437539880 & $7.091550 e^{-14}$ \\
0.7 & -0.148975591137422590 & -0.148975591137365140 & $5.789813 e^{-14}$ \\
0.8 & -0.089865792823444049 & -0.089865792823403012 & $4.170275 e^{-14}$ \\
0.9 & -0.040656965974059656 & -0.040656965974037895 & $2.220446 e^{-14}$ \\
1.0 & 0.000000000000000000 & -0.000000000000000444 & $4.441233 e^{-17}$ \\
\hline
\end{tabular}

Table 3: Numerical results of the new method, solving Problem with $h=0.01$

[9] H. A. Emad,E. Abdelhalim and R. Randolph, Advances in the Adomian decomposition method for solving two-point nonlinear boundary value problems with Neumann boundary conditions, Computer and Mathematics with Applications, 63,2012,pp. 1056-1065

[10] Z. A. Majid, M.M. Hasni \& N. Senu, Solving second order linear Dirichlet and Neumann boundary value problems by block method. Int J Appl Math, 43,(2),(2013), 71-76. 
